\title{
Neurotransmitter changes during interference task in anterior cingulate cortex: evidence from fMRI-guided functional MRS at $3 \mathrm{~T}$
}

\author{
Simone Kühn ${ }^{1,4} \cdot$ Florian Schubert $^{2} \cdot$ Ralf Mekle $^{2} \cdot$ Elisabeth Wenger $^{1}$ • \\ Bernd Ittermann $^{2} \cdot$ Ulman Lindenberger $^{1} \cdot$ Jürgen Gallinat $^{3,4}$
}

Received: 20 January 2015/Accepted: 4 May 2015/Published online: 15 May 2015

(c) Springer-Verlag Berlin Heidelberg 2015

\begin{abstract}
Neural activity as indirectly observed in blood oxygenation level-dependent (BOLD) response is thought to reflect changes in neurotransmitter flux. In this study, we used fMRI-guided functional magnetic resonance spectroscopy (MRS) to measure metabolite/BOLD associations during a cognitive task at $3 \mathrm{~T}$. GABA and glutamate concentration in anterior cingulate cortex (ACC) were determined by means of MRS using the SPECIAL pulse sequence before, during and after the performance of a manual Stroop task. MRS voxel positions were centred around individuals' BOLD activity during Stroop performance. Levels of GABA and glutamate showed inverted U-shape patterns across measurement time points (before, during, and after task), glutamine increased linearly and total creatine did not change. The GABA increase during task performance was associated with ACC BOLD signal changes in both congruent and incongruent Stroop conditions. Using an fMRI-guided MRS approach, an association between induced inhibitory neurotransmitter increase and BOLD changes was observed. The proposed procedure might allow the in vivo investigation of normal
\end{abstract}

Simone Kühn

kuehn@mpib-berlin.mpg.de

1 Max Planck Institute for Human Development, Center for Lifespan Psychology, Lentzeallee 94, 14195 Berlin, Germany

2 Physikalisch-Technische Bundesanstalt (PTB), Abbestr. 2-12, 10587 Berlin, Germany

3 St. Hedwig-Krankenhaus, Clinic for Psychiatry and Psychotherapy, Charité University Medicine, Große Hamburger Straße 5-11, 10115 Berlin, Germany

4 University Clinic Hamburg-Eppendorf, Clinic and Policlinic for Psychiatry and Psychotherapy, Martinistraße 52, 20246 Hamburg, Germany and dysfunctional associations between neurotransmitters and BOLD signal crucial for cerebral functioning.

Keywords Spectroscopy $\cdot$ GABA glutamate $\cdot$ MRS · fMRI $\cdot$ Stroop

Neurotransmitters are commonly classified as excitatory or inhibitory, although this property is, strictly speaking, not an attribute of the neurotransmitter itself but depends on the properties of the receptors that the neurotransmitters bind to on the postsynaptic cell. The majority of cortical neurons are excitatory glutamatergic cells, the remaining are inhibitory gamma-aminobutyric acid (GABA)ergic interneurons (Markram et al. 2004). The coordination between these glutamatergic excitatory neurons and GABAergic interneurons has been described as fundamental to the regulation of neural activity and is the target for several pharmacological interventions (Wang 2010).

In addition to magnetic resonance spectroscopy (MRS) methods dedicated to the detection of specific neurotransmitters, such as glutamate (Schubert et al. 2004) and GABA (Rothman et al. 1993), both these neurotransmitters as well as even glutamine can also be detected and fairly reliably quantified in the human brain by means of ultrashort-echo time MRS (Mlynarik et al. 2006; Mekle et al. 2009). Recent studies have discovered a link between resting GABA levels and the blood oxygen level-dependent (BOLD) functional magnetic resonance imaging (fMRI) signal (Donahue et al. 2010; Muthukumaraswamy et al. 2009; Northoff et al. 2007; Arrubla et al. 2014), for an overview see (Duncan et al. 2014). For instance, Northoff et al. (2007) reported a positive correlation between GABA level and BOLD signal in anterior cingulate cortex (ACC) at rest, whereas Donahue et al. (2010) as well as 
Muthukumaraswamy et al. (2009) observed a negative correlation between resting GABA in visual cortex and BOLD signal during checkerboard stimulation.

In contrast to the frequent assessment of metabolite concentrations at rest, dynamics of GABA and glutamate during any kind of task performance have only seldomly been investigated. Such studies include are reports on GABA reductions in the motor system (M1) in association with motor learning (Floyer-Lea et al. 2006), transcranial direct current stimulation (Stagg et al. 2011) and transcranial magnetic stimulation (Stagg et al. 2009a), as well as reports on GABA increases after continuous theta burst stimulation, which is known to have an inhibitory effect on the brain (Stagg et al. 2009b). To the best of our knowledge, so far only one study has focused on the association of changes in neurotransmitter concentrations and haemodynamics in a cognitive task, with the topic of working memory (Michels et al. 2012). An initial increase of GABA in dorsolateral prefrontal cortex (DLPFC) was observed during the first working memory run, followed by a continuous decrease across subsequent task runs. Overall a negative association between GABA level at rest and perfusion during task performance was reported.

We set out to explore the association between the neurotransmitters GABA and glutamate and BOLD activity in the ACC during conflict processing. The ACC is known to be involved in conflict monitoring; it detects conflict between simultaneously active, competing representations and subsequently engages the DLPFC to resolve this conflict (Botvinick et al. 2004; Carter et al. 1998; Carter and van Veen 2007). To serve its monitoring function, the ACC signal needs to be dynamically adjusted, depending upon the current level of conflict. We examined whether dynamic ACC recruitment in response to conflict is related to inhibitory (i.e. GABAergic) neurotransmission, excitatory (i.e. glutamatergic) neurotransmission, or both. To this end, we used a manual version of the Stroop task, during which participants responded to the print colour of colour words (Stroop 1935). Whenever the print colour fits the colour word (e.g. "blue" printed in blue colour), participants are faster and more accurate, because print colour and colour word are mapped onto the same response. However, when print colour is different from colour word (e.g. "blue" printed in red colour), response times are slowed and an increased number of errors occur, as print colour and the colour word activate different response options and therefore induce conflict. Quantitative meta-analyses have shown that the comparison of incongruent (e.g. "blue" printed in red) and congruent (e.g. "blue" printed in blue) Stroop trials in fMRI results in reliable ACC activation across multiple independent studies (Laird et al. 2005; Nee et al. 2007). To achieve optimal positioning of the MRS voxel, we first acquired fMRI data, while participants performed the Stroop task. We then used the individual peak activation together with the group-level random effect activation within ACC during incongruent vs. congruent trials to position the MRS voxel accordingly for a subsequent session, during which neurotransmitters were quantified.

The measurement of neurotransmitter concentrations was performed before, during and after the cognitive task performance to investigate the complete dynamics over the course of the task session. Our main hypotheses were that GABA, glutamate and glutamine concentrations in ACC would increase from pre-task to during-task measurement. As for post-task measurements, a decrease in all neurotransmitter concentrations with respect to during-task was expected. In addition, we expected an association between task-related neurotransmitter change and task-related BOLD activity. Methodologically, we expected that the detection of an association between transmitter and brain activity would be facilitated by the activation-guided positioning of the MRS voxel.

\section{Methods}

\section{Participants}

Nineteen healthy students (11 female and 8 male) with a mean age of 25 years $(\mathrm{SD}=1.7)$ participated on the basis of informed consent. The study was conducted according to the Declaration of Helsinki, with approval of the local ethics committee. All subjects had normal or corrected-tonormal vision. No subject had a history of neurological, major medical, or psychiatric disorder, were medication free and non-smokers. All participants were right-handed as assessed by the Edinburgh handedness questionnaire (Oldfield 1971).

\section{Procedure}

Participants took part in two separate scanning sessions, one consisting of an fMRI measurement, the other consisting of MRS measurements. In both sessions, they completed a manual version of the Stroop task of 13 min duration. The colour words "green", "blue", "yellow" and "red" (in German) were presented randomly in these four different colours. Participants were instructed to press buttons to indicate the print colour of the presented word. Each trial consisted of the presentation of one of the colour words for $3 \mathrm{~s}$. Stimulus presentations were separated by a jitter interval of 5-6.5 s (varied in steps of $500 \mathrm{~ms}$ ). The task consisted of 120 trials. Half of the trials were congruent trials, where the print colour was congruent to the colour word (e.g. "blue" in blue colour), the other half 
were colour words presented in an incongruent print colour (e.g. "blue" in red colour).

In a separate scanning session, 2 to 5 days after the fMRI acquisition to enable the individual analysis of the fMRI data, MRS data were acquired. During the first MRS acquisition participants rested, during the next acquisition, the Stroop task was administered for the entire duration of the measurement. After this, another MRS acquisition at rest was performed.

\section{Scanning procedure}

All MR data were collected on a 3T Verio MRI scanner system (Siemens Healthcare, Erlangen, Germany) using a 32-channel receive-only head coil. First, high-resolution anatomical images were acquired using a three-dimensional T1-weighted magnetization-prepared gradient-echo sequence (MPRAGE), repetition time $=2.3 \mathrm{~ms}$; echo time $=3.03 \mathrm{~ms}$; flip angle $=9 ; 256 \times 256 \times 192$ matrix , $1 \times 1 \times 1 \mathrm{~mm}^{3}$ voxel size. Whole-brain functional images were collected on the same scanner using a T2*-weighted EPI sequence sensitive to BOLD contrast $(T R=2000 \mathrm{~ms}$, $\mathrm{TE}=30 \mathrm{~ms}$, image matrix $=64 \times 64, \mathrm{FOV}=224 \mathrm{~mm}$, flip angle $=80^{\circ}$, slice thickness $=3.5 \mathrm{~mm}, 35$ near-axial slices, aligned with the AC/PC line).

For MRS, all first- and second-order shims were adjusted using FAST(EST)MAP. Spectra were acquired at rest, during Stroop task administration and at rest again, using the spin-echo full intensity-acquired localized (SPECIAL) technique (Mlynarik et al. 2006). The SPECIAL sequence is based on a combination of 1D imageselected in vivo spectroscopy (ISIS) (Ordidge 1986) and a slice-selective spin-echo sequence. Localization in the direction perpendicular to the plane selected by the spin echo is achieved by application of a slice-selective adiabatic inversion pulse in alternate scans. Then a spin-echo sequence is applied using an asymmetric $90^{\circ}$ and a $180^{\circ}$ slice-selective refocusing pulse to localize the signal within two dimensions. The signal is added in odd scans and subtracted in even scans. Since only one refocusing pulse is used, very short echo times can be achieved using this technique (Mekle et al. 2009). Spectra were recorded from a voxel of $25 \times 35 \times 20 \mathrm{~mm}^{3}$ placed around the region of maximum activation in ACC during Stroop task performance in native space. For each metabolite spectrum, 256 scans were acquired using $\mathrm{TR}=3 \mathrm{~s}$ and $\mathrm{TE}=8.5 \mathrm{~ms}$. Immediately afterwards, a spectrum without water suppression was recorded ( 8 averages).

\section{fMRI data pre-processing and main analysis}

The fMRI data were analysed using the SPM8 software (Wellcome Department of Cognitive Neurology, London,
UK). The first 4 volumes of all EPI series were excluded from the analysis to allow the magnetisation to reach a dynamic equilibrium. Data processing started with slice time correction and realignment of the EPI datasets. A mean image for all EPI volumes was created, to which individual volumes were spatially realigned by means of rigid body transformations. The T1-weighted structural image was co-registered with the mean image of the EPI series. Then the structural image was normalized to the Montreal Neurological Institute (MNI) template for the random effects analysis. The normalization parameters were then applied to the EPI images to ensure an anatomically informed normalization. A commonly applied filter of $8 \mathrm{~mm}$ FWHM (full-width at half maximum) was used. Low-frequency drifts in the time domain were removed by modelling the time series for each voxel by a set of discrete cosine functions to which a cutoff of $128 \mathrm{~s}$ was applied. The statistical analyses were performed using the general linear model (GLM). We modelled each trial as an event separately for the conditions congruent and incongruent. These vectors were convolved with a canonical haemodynamic response function (HRF) and its temporal derivatives to form regressors in a design matrix. Furthermore, six movement regressors were entered into the GLM. The parameters of the result.ing general linear model were estimated in the standard way and used to form contrasts, testing for main effects and interactions. The resulting contrast image was then entered into one sample $\mathrm{T}$ tests at the second (between-subject) level. To display the results of the group analysis, statistical values were thresholded with a level of significance of $p<0.001$ ( $z>3.09$, uncorrected); a significant effect is reported when the volume of the cluster was greater than the minimum cluster size determined by Monte Carlo simulation above which the probability of type I error was $<0.05$ (Song et al. 2011).

The same analysis was conducted in native space (without the normalization step) to guide MRS-voxel positioning. The voxel position was guided by the individuals' brain activity. The subjects' voxel position included the individual activation within the ACC as well as the cluster identified by means of a random effects group analysis on the contrast incongruent $>$ congruent trials (Fig. 1).

\section{Magnetic resonance spectroscopy (MRS) processing}

Only data, for which the linewidth of the water signal after shimming was $<7 \mathrm{~Hz}$, were considered for further analysis. This was the case for all but one subject, which reduced the sample size to 18. Metabolite levels were determined using LCModel (Provencher 1993) with a simulated basis set, applying the unsuppressed water scan for eddy current 


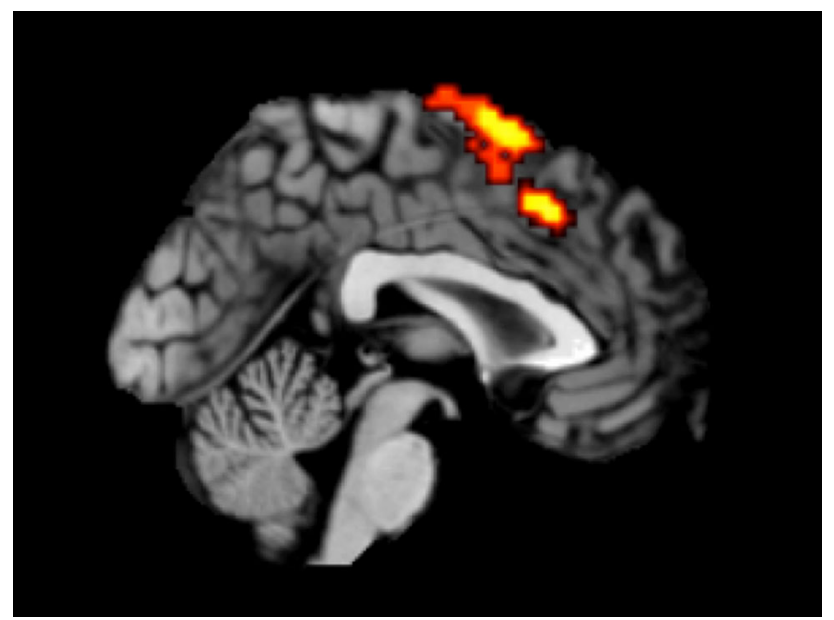

Fig. 1 Main contrast of incongruent vs. congruent Stroop trials in fMRI across the group of subjects (peak voxel: 6, 18, 42) mapped onto a template in MNI space (colin27)

correction and referencing. From segmentation of the T1weighted images using SPM8 (http://www.fil.ion.ucl.ac.uk/ $\mathrm{spm} /$ software/spm8/), mean fractions of grey matter, white matter and cerebrospinal fluid (CSF) in the spectroscopic voxel of 0.56:0.265:0.175 were deduced and used to calculate the appropriate water reference parameters for the respective LCModel control file. MRS data were expressed as fractional metabolic change score with the reference being the first resting-state MRS measurement.

\section{Results}

\section{Behavioural data}

Behavioural data obtained during the fMRI session revealed a clear Stroop effect, namely longer reaction times in the incongruent (mean $1175 \mathrm{~ms}$ ) compared with the congruent $(1029 \mathrm{~ms})$ condition $[t(17)=3.673, p<0.01]$. Furthermore, participants committed more errors for the incongruent (mean 2.39) than for the congruent (1.50) condition, an effect that was only marginally significant $[t(17)=2.082, p=0.053]$. The behavioural data obtained during the MRS session likewise revealed an interference effect in reaction times [congruent: $874 \mathrm{~ms}$, incongruent: $1049 \mathrm{~ms} ; t(17)=4.444, p<0.001$ ], but no significant effects in errors [congruent: 2.44 , incongruent: $3.00 ; t(17)=1.097, p=0.288]$. The Stroop effect in the reaction times and in the errors was correlated between the fMRI and the MRS session [RT: $r(18)=.52$, $p<0.05$; errors: $r(18)=.47, p<0.05$ ]. When averaging the Stroop effect in the error rate across the fMRI and MRS sessions the overall effect was significant $[t(17)=2.15, p<0.05]$.

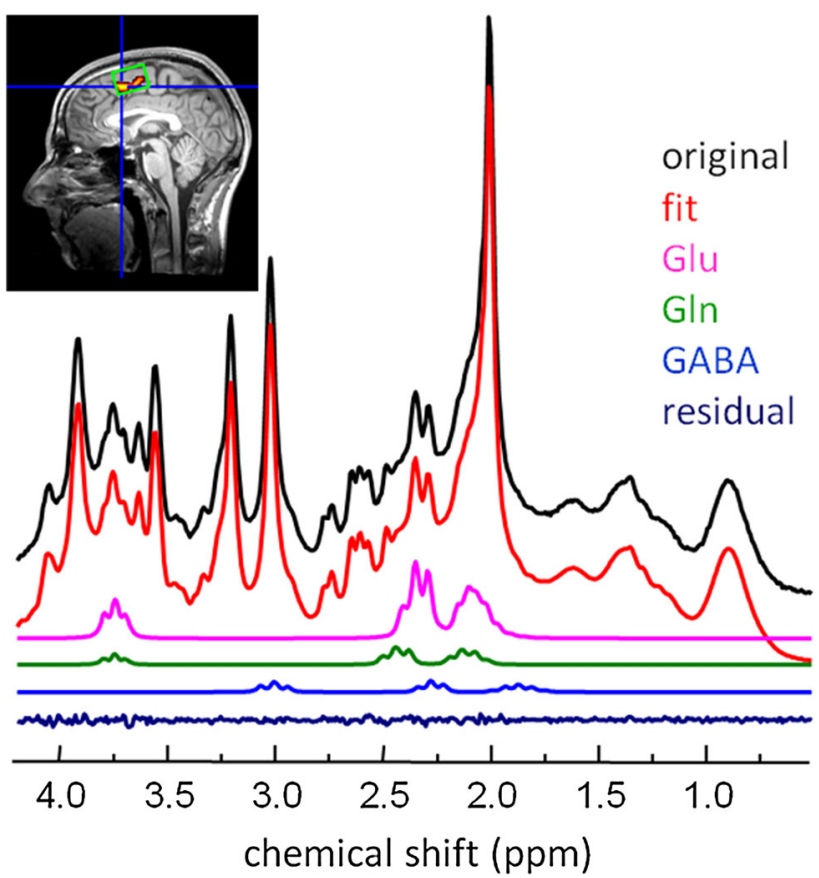

Fig. 2 SPECIAL spectrum, LCModel fits for glutamate (Glu), glutamine (Gln) and GABA, and residual from Stroop-activated anterior cingulate cortex (ACC) voxel. TE $=8.5 \mathrm{~ms}, \mathrm{TR}=3 \mathrm{~s}$, number of acquisitions: 256. Top left The MRS voxel in the ACC is fine-positioned according to the BOLD activation profile of the individual participant

\section{fMRI data}

The results of the random effects fMRI analysis of the contrast incongruent $>$ congruent are depicted in Fig. 1 . Apart from a cluster in $\operatorname{ACC}(6,18,42)$ additional clusters were observed in bilateral inferior frontal gyrus (IFG) extending into bilateral insula (left IFG: $-54,33,3$ and -54 , 15, 24; right IFG: 45, 27-3).

In a separate session-after the analysis of the fMRI data-MRS was acquired using voxel positions guided by the individually localized brain activity within ACC (Fig. 2).

\section{MRS neurotransmitter data}

The mean ( \pm standard deviation) water linewidth was calculated from a fit to the water spectrum after shimming to be $(6.2 \pm 0.5) \mathrm{Hz}$. The excellent quality of the acquired metabolite spectra is exemplified in Fig. 2, which includes the overall fit and the fit results for GABA, glutamate and glutamine. The average signal-to-noise ratio (SNR) as calculated by LCModel, where it is defined as the ratio of the maximum in the spectrum-minus-baseline over the analysis window, i.e. the NAA peak at $2.01 \mathrm{ppm}$, to twice the root-mean-square of the fit residual, was $144 \pm 13$. In addition, since SNR calculated by LCModel might depend 
Table 1 Mean raw metabolite concentrations in mmol/l (SD) during the three experimental phases

\begin{tabular}{lrrr}
\hline & \multicolumn{1}{c}{ Pre-task } & During task & \multicolumn{1}{c}{ Post-task } \\
\hline GABA & $0.72(0.19)$ & $0.88(0.20)$ & $0.81(0.22)$ \\
Glutamate & $6.54(0.78)$ & $6.81(0.62)$ & $6.48(0.60)$ \\
Glutamine & $1.22(0.15)$ & $1.31(0.34)$ & $1.34(0.28)$ \\
Total creatine & $7.25(0.61)$ & $7.40(0.40)$ & $7.28(0.49)$ \\
NAA & $10.43(0.90)$ & $10.82(0.60)$ & $10.57(0.84)$ \\
\hline
\end{tabular}

on fit quality, and to provide another assessment of MRS data quality, SNR of the spectra was also computed by dividing the height of the NAA peak by the standard deviation of the noise in the peak-free region of 6-8 ppm. The corresponding result was $576 \pm 82$. The mean neurotransmitter concentrations for the three test periods together with that for total creatine are shown in Table 1. Note that the values are those returned by LCModel analysis, i.e. they are not corrected for relaxation and CSF content of the spectroscopic voxel, which was not required, because we compared the values within the same session. If, however, these corrections are applied (using the mean CSF fraction of 0.175 and published relaxation times at $3 \mathrm{~T}$ (Schubert et al. 2004; Edden et al. 2012), concentrations agree with published values (Govindaraju et al. 2000). Mean Cramér-Rao lower bounds (CRLB) were for GABA $16.5 \%$, glutamate $4.1 \%$, glutamine $12.2 \%$ and total creatine $1.1 \%$. Of the 54 GABA CRLBs, 5 were between 20 and $25 \%$, all others below $20 \%$. All GABA values were included in the data analysis. To investigate associations between transmitters and brain activity, we extracted BOLD percent signal changes during the fMRI session from the location where the MRS voxels were positioned in the second session. When comparing fMRI activation during congruent with the incongruent condition, we found - in line with our positioning procedure-higher activity during incongruent compared to congruent trials $[t(17)=3.484, p<0.01]$ within the voxel.

By means of repeated measures ANOVA with the factor time (pre- vs. during vs. after task), we explored variations of metabolite concentrations in ACC over time. GABA [quadratic: $\quad F(1,17)=6.705, \quad p=0.019$ ], glutamate [quadratic: $F(1,17)=7.069, p=0.017$ ] and glutamine [linear: $F(1,17)=13.390, p=0.002$ ] showed a significant effect of time. Testing the effect on metabolite ratios (/total creatine) yielded for GABA $p=0.049$ [quadratic: $F(2,34)=3.308$ ], for glutamate $p=0.035$ [quadratic: $F(2,34)=3.7$ ] and for glutamine $p=0.24$ [linear: $F(2,34)=1.449]$. Thus, GABA and glutamate increased during task performance and decreased again, whereas glutamine increased during the task and continued to increase after the task (this effect being discernible only for

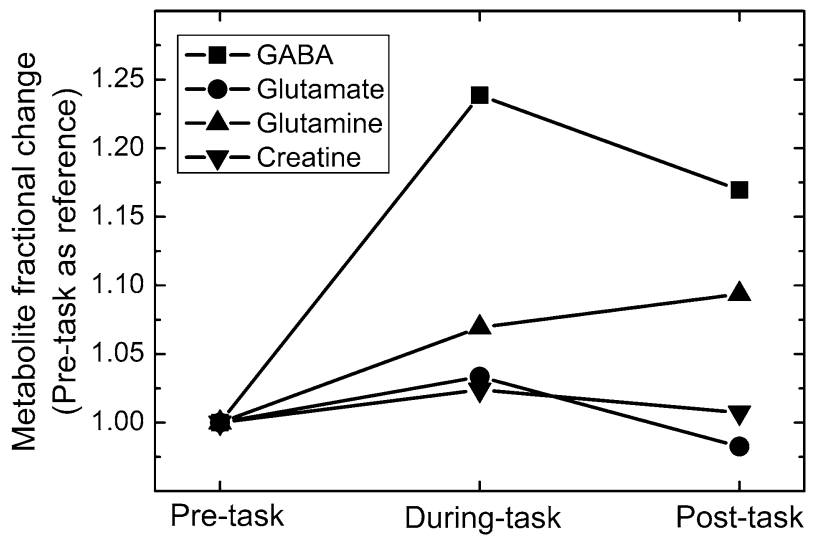

Fig. 3 Metabolite fractional change in relation to the respective metabolite measured during the first resting MRS (pre-task)

the water-referenced concentrations). Neither total creatine $(p=0.32)$ nor NAA $(p=0.11)$, showed a significant time effect. In Fig. 3, we present metabolite changes as percent changes from the initial, pre-task measurement. When testing the increase during task performance by means of paired samples $t$ test, only the GABA increase reached significance $[t(17)=-4.664, p<0.001$; for glutamate, glutamine, creatine $p>0.182]$.

\section{Association between GABA change and fMRI activity}

Given that the GABA increase during task performance reached statistical significance in post hoc $t$ tests, we associated the increase between the pre-task MRS and the task MRS with the BOLD percent signal change within the ACC voxel obtained during the first session in: (a) the congruent Stroop condition (e.g. "blue" printed in blue colour); (b) the incongruent Stroop condition (e.g. "blue" printed in red colour); and (c) the difference between the incongruent and congruent condition as an indicator of task-associated within-person adjustments. We found a significant negative correlation between GABA change during task and BOLD signal change during the congruent Stroop condition $[r(18)=-0.522, p=0.026$, Fig. 4]; Likewise a negative correlation with the incongruent Stroop condition was observed $[r(18)=-0.475$, $p=0.046$, Fig. 4], congruent did not correlate significantly with task-related GABA change $[r(18)=0.306$, $p=0.218]$. In contrast, for the glutamate increase, we found no significant association to BOLD signal [congruent condition: $r(18)=-0.270, p=0.279$, incongruent condition: $r(18)=-0.241, p=0.336$; incongruent minus congruent condition: $r(18)=0.154, p=0.542$ ].

For bilateral inferior frontal gyrus, brain regions that were also activated in the contrast incongruent vs. 

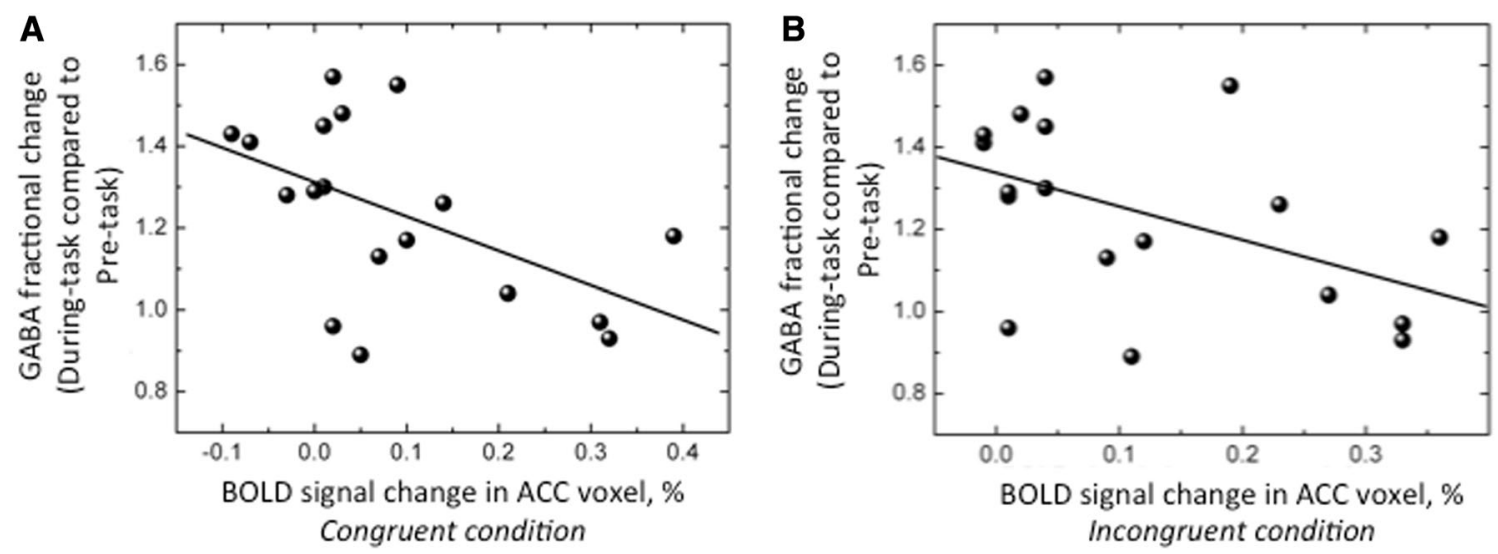

Fig. 4 Scatterplot of GABA percent changes (during-task compared to pre-task) and BOLD signal change in the a congruent condition and b incongruent condition

congruent same as ACC, we did not find a significant association, neither for GABA change [congruent condition: $r(18)=-0.169, \quad p=0.502, \quad$ incongruent condition: $r(18)=-0.352, p=0.152$; incongruent minus congruent condition: $r(18)=0.056, p=0.825$ ] nor for glutamate change [congruent condition: $r(18)=0.144, p=0.569$, incongruent condition: $r(18)=0.172, p=0.494$; incongruent minus congruent condition: $r(18)=0.115$, $p=0.651]$, showing the specificity of the observed results for the brain region where GABA was measured.

\section{Discussion}

The present study demonstrates a modulation of neurotransmitters across a complete cognitive task session, namely from pre-task, during task up to post-task time points. GABA and glutamate showed an inverted U-shape pattern with an increase during task performance and a drop after task performance. In contrast, glutamine showed a linear increase. Total creatine did not change over time. The significant changes of the metabolite levels with time were shown not only for the water-referenced values of GABA and glutamate but also for the data normalized to total creatine. This indicates that these changes are unlikely to be due to head displacement during the (rather long) MRS acquisition time, which might have changed the metabolite level/water ratios, but are rather due to true concentration variation. However, the increase in glutamine over time could not be confirmed in this manner. This may be due to the moderate intrinsic precision of the glutamine measurement or could be related to limited numerical precision, when dividing small concentration changes by an overall constant, but numerically slightly varying value.
Our result of an increase in glutamate during functional activation confirms the findings of previous reports on functional 1H-MR spectroscopy in the visual cortex (Mangia et al. 2007; Schaller et al. 2013) or the motor cortex (Schaller et al. 2014), applying appropriate paradigms.

A recent meta-analysis has demonstrated trait-like changes in glutamate level in major depression. Patients were shown to have lower levels of glutamate and Glx (the composite measure of glutamate and glutamine) compared with controls (Luykx et al. 2012). Interestingly, Glx seems to be decreased during current episodes, supporting our present observation that the glutamate-associated metabolite levels can change over short periods of time. Moreover, the fact that patients suffering from major depression consistently show reductions in Stroop performance (Epp et al. 2012) fits well to our observation that Stroop performance is accompanied by glutamate increases during the task.

Within the context of the above-mentioned previous studies focusing on metabolite level changes during visual stimulation the study by (Lin et al. 2012), who used MRS at $7 \mathrm{~T}$, reported a change in cortical GABA, namely an increase by about $5 \%$, which was evoked by visual stimulation, but did not reach significance. Very recently, we observed a significant GABA decrease by $9 \%$ on average $(p<0.01)$ during a visual paradigm probing retinotopy using SPECIAL on a 7-T scanner (Mekle 2014). Measuring at $7 \mathrm{~T}$ as well, neither of the other studies mentioned above (Mangia et al. 2007; Schaller et al. 2013; 2014) could detect changes in GABA levels upon functional activation. Even so, it should be noted that other investigations indeed suggest changes in GABA concentration to occur as a result of functional activation, e.g. in sensorimotor cortex during motor learning (Floyer-Lea 
et al. 2006) and in frontal brain regions during working memory (Michels et al. 2012). Nota bene both increases and decreases in GABA levels were observed. This raises the question whether GABA can be determined using short-echo time MRS at $3 \mathrm{~T}$ reliably enough to draw conclusions on the effects of functional stimulation. Floyer-Lea et al. (2006) and Michels et al. (2012) used an alternative method for GABA, the MEGA-edited MRS technique (Mescher et al. 1998), which, while suffering from some disadvantages such as low intrinsic signal and low number of detectable metabolites, is often considered the gold standard for GABA measurement because of its high selectivity achieved by spectral editing. In contrast, short-echo time MRS sequences allow a large number of metabolites to be detected with high precision due to minimal transverse relaxation and spin evolution effects and high SNR (Mekle et al. 2009; Tkac et al. 2009), which have been the reasons for using such a method in the present study. Besides yielding a much higher signal-tonoise ratio than MEGA-edited GABA spectroscopy due to the shorter echo time and the low editing efficiency of the latter, SPECIAL is advantageous, since it includes the three methylene resonances of GABA to estimate its intensity instead of only one as in MEGA-editing. On the other hand, SPECIAL lacks selectivity for a specific compound like GABA and is characterized by strong background contribution due to unrelaxed macromolecule resonances. In contrast to single-shot MRS localization techniques, such as STEAM or PRESS, full localization in SPECIAL is achieved using two (subsequent) acquisitions. As a consequence, this scheme is slightly more sensitive with respect to motion, frequency drift or any other scanner instability that might occur during these two acquisitions. This holds for all two-shot methods including editing sequences, such as MEGA-PRESS. In this study with healthy volunteers though, an increased level of artefacts due to such effects was not observed. An extensive study on GABA quantification using short TE at $3 \mathrm{~T}$ was conducted by Near et al. (2013), who compared short-echo time SPECIAL as employed in the present study with MEGA-edited MRS. GABA levels quantified by both methods were correlated with $r=0.58$, which was significant $(p<0.05)$. For GABA concentrations estimated using SPECIAL in 500 simulated spectra generated with experimental conditions similar to the ones of the in vivo data acquired in this study, the correlation coefficient with the actual values was as high as 0.84 . Although the absolute concentration of GABA was systematically underestimated using SPECIAL, the reproducibility was shown to be approximately the same for both methods. The authors of this in-depth comparison concluded that when similar spectral quality is ensured across datasets and data not fulfilling linewidth and SNR criteria are excluded, short-echo time SPECIAL is "capable of providing reproducible GABA measurements". Their volume of interest was restricted to the occipital cortex, which is a region where very high field homogeneity and SNR can be obtained. This is comparable to our spectroscopic voxel where careful shimming achieved high spectral dispersion/narrow linewidths, and the SNR of the acquired data was high throughout the sessions. Moreover, our experimental setup does not necessarily require absolute GABA concentrations to be correctly measured, since for a follow-up measurement in a subject during a single session the evolution of a relative signal is sufficient and significant. We conclude that, even if methods like edited spectroscopy at $3 \mathrm{~T}$ or short-echo time spectroscopy at ultrahigh field may be superior to SPECIAL at $3 \mathrm{~T}$ for GABA quantification, the GABA time course reported here represents a true evolution of the concentration of this neurotransmitter. However, since these results are novel and not undisputable with regard to other functional MRS studies, they should be considered preliminary.

In this study, GABA was significantly increased by $22 \%$ from pre-task to task (see Table 1). In the only other study that attempted to assess neurotransmitter changes during a cognitive task (Michels et al. 2012), a fractional increase of GABA of roughly $6 \%$ during the initial task was reported, which was also significant, but clearly smaller in magnitude than the result from this study. However, in general, it is rather difficult to predict the magnitude of neurotransmitter changes in cognitive experiments as measured by MRS. The experimental paradigm, selection of subjects, data acquisition including magnetic field strength, and the particular neurotransmitter of interest can all influence the observed outcome. Hence, to assess neurotransmitter changes in a study, it is required that the sensitivity of the applied measurement technique has to be sufficiently high to detect the induced neurotransmitter change, which can vary in magnitude, that ought to take place during the course of the experiment.

GABA has recently gained increasing attention in schizophrenia. However, pertinent findings have been inconsistent so far. Some studies found reductions in GABA levels (Goto et al. 2009; Yoon et al. 2010), whereas others reported increases (Yoon et al. 2010; Kegeles et al. 2012) and another did not find any differences (Rowland et al. 2013), but a trend for reductions of GABA in ACC in older schizophrenic subjects. Interestingly, the authors found an association between performance in an attention-demanding task similar to digit symbol and GABA levels with poorer performance associated with lower GABA levels. This finding may be seen as in line with our observation that Stroop as an interference task is accompanied by GABA increases.

The increase in GABA was negatively associated with BOLD signal change in the congruent and incongruent 
condition assessed in a separate session, suggesting an association between GABA and the dynamics of brain activation that is stable across time.

The present association between GABA and glutamate changes, on the one hand, and the course of functional task activation, on the other, raises questions of causality. The fact that the time course of the metabolite concentrations varies with the three paradigm phases, and in particular the reversible nature of the changes in glutamate and GABA, together with unchanged total creatine, suggests a causal link between neural activation as assessed with the BOLD signal, and neurotransmitter concentrations as assessed by MRS.

The peak coordinate of the random effects analysis of incongruent vs. congruent Stroop condition $(6,18,42)$ was located in close proximity to the coordinates identified in quantitative meta-analyses on Stroop task fMRI data [MNI coordinate: 3, 14, 45 (Laird et al. 2005); MNI coordinate: $0,20,40$ (Nee et al. 2007)]. To improve positioning of the ACC voxel beyond what is known from previous studies, we determined each participant's activation peak. Then, the voxel was positioned such that it encompassed both the individual's activation and the activation ascertained by group random effects. This procedure allows the direct association of personalized brain activation pattern with underlying neurochemistry.

The quadratic time effect was significant for GABA and glutamate. A significant increase from pre-task to task was found for GABA only. This result clearly differs from the reported GABA decreases in motor cortex (Floyer-Lea et al. 2006; Stagg et al. 2009a, 2011), but can be seen in agreement with the initial GABA increase in a study exploring the effects of a working memory task on neurotransmitter levels (Michels et al. 2012). The present MRS acquisition and simultaneous task performance was of about 13 min duration, comparable to the first acquisition in (Michels et al. 2012) that took $10 \mathrm{~min}$. Future research should therefore address the question whether GABA levels decrease over longer periods of Stroop task performance after the observed initial increase. While the total creatine concentration did not change across the task performance, a simultaneous tendency for an increase of GABA, glutamine and glutamate was observed in (Patel et al. 2005) suggesting a general up-regulation of excitatory and inhibitory neurotransmitters. The observation that glutamate tends to decrease after the task, whereas glutamine keeps rising underlines the biological plausibility of the observed effect. According to the glutamate/glutamine cycle (Schousboe et al. 1993), glutamate is cleared from the synaptic space and internalized into astrocytes, where it is converted to glutamine resulting in rising glutamine concentrations.

Over and above the task-related changes in neurotransmitter concentrations, we found a negative correlation between GABA increase and BOLD signal during congruent as well as incongruent Stroop trials compared to baseline, but not with glutamate change. The observation of this association extends previous knowledge of correlations between baseline GABA levels and fMRI task-related activity (Donahue et al. 2010; Muthukumaraswamy et al. 2009; Stagg et al. 2011). Interestingly, this association was specific for BOLD measured in ACC and not present in bilateral inferior frontal gyrus, although this area was also active in the contrast of interest.

We did not observe a correlation between task-related changes in GABA concentrations and the difference of BOLD signals between incongruent and congruent conditions. This result speaks against a major role of inhibitory neurotransmission in conflict-related processing of the ACC. However, given the small sample size and the generally low reliability of difference scores, this null result must be interpreted with great caution. One may speculate that other neurotransmitters or neuromodulators may be more relevant in this context. Particularly in prefrontal areas, the dopamine system mediates activation in general (Winterer and Weinberger 2004) and conflict processing in particular (Guitart-Masip et al. 2014; Moeller et al. 2014; Onur et al. 2011).

The present findings may be potentially relevant in the context of clinical studies. Although GABA is still challenging to measure and therefore has not been as frequently assessed in psychiatric disease as other metabolites, such as NAA and glutamate, GABA reductions compared to healthy controls have been demonstrated in bipolar disorder (Bhagwagar et al. 2008) and in major depression (Hasler et al. 2007; Sanacora et al. 1999, 2000, 2004). For insomnia, GABA reductions (Plante et al. 2012) as well as increases have been reported (Morgan et al. 2012) and GABA increases have been reported in post-traumatic stress disorder (Meyerhoff et al. 2014; Michels et al. 2014; Rosso et al. 2014).

Most interestingly from a functional perspective, GABA levels in major depression have been shown to be renormalized after therapy with selective serotonin reuptake inhibitors (SSRIs) (Sanacora et al. 2002) and after electroconvulsive therapy (Sanacora et al. 2003). A more statelike view on MRS measurements, compared to the more common trait-like approach usually employed by research in the field of psychiatry, may help to make better use of MRS in the prediction of therapy outcome. One may attempt to compare GABA levels in major depression with and without a single dose of SSRIs, e.g. to predict longterm treatment outcome.

Replication of our findings regarding the functional changes in GABA using other MRS techniques including MEGA-editing and MRS at $7 \mathrm{~T}$ would be most enlightening. 


\section{Further considerations}

MRS has been shown to allow accurate quantification of the concentration of neurochemicals within defined areas of the brain. However, it does not provide direct information on activity at the synapses. What we measure in the present study is an estimate of bulk metabolite levels in a fairly large voxel, therefore only a fraction of the GABA and glutamate measured is actually located in the synaptic cleft. Furthermore, a number of additional neuromodulators, such as dopamine, serotonin, and acetylcholine, could be relevant in conflict processing (Voon et al. 2010). Since $1 \mathrm{H}$ MRS is relatively insensitive to changes in these chemicals, we may have missed processes that could potentially correlate with conflict-related BOLD modulation in ACC. Furthermore, the fact that we have determined the voxel position according to each individuals' BOLD activation allowed for variation in terms of the anatomical location across participants. An additional MRS voxel in a task-unrelated brain region would help to demonstrate the specificity of the observed effects.

As for the question of causality between Stroop activation and neurotransmitter concentrations, the present findings need to be followed up by future research with bigger sample sizes. For instance, it seems worthwhile exploring MRS across Stroop task blocks that vary in the degree of conflict and an additional "baseline" block without a task to further elucidate the dynamics of neurotransmitter changes and their association to brain activation.

\section{References}

Arrubla J, Tse DH, Amkreutz C, Neuner I, Shah NJ (2014) GABA concentration in posterior cingulate cortex predicts putamen response during resting state fMRI. PLoS One 9(9):e106609. doi:10.1371/journal.pone.0106609

Bhagwagar Z, Wylezinska M, Jezzard P, Evans J, Boorman E, Matthews MP, Cowen JP (2008) Low GABA concentrations in occipital cortex and anterior cingulate cortex in medication-free, recovered depressed patients. Int J Neuropsychopharmacol Off Sci J Coll Int Neuropsychopharmacol 11(2):255-260. doi:10. 1017/S1461145707007924

Botvinick MM, Cohen JD, Carter CS (2004) Conflict monitoring and anterior cingulate cortex: an update. Trends Cogn Sci 8(12):539-546. doi:10.1016/j.tics.2004.10.003

Carter CS, van Veen V (2007) Anterior cingulate cortex and conflict detection: an update of theory and data. Cogn Aff Behav Neurosci 7(4):367-379

Carter CS, Braver TS, Barch DM, Botvinick MM, Noll D, Cohen JD (1998) Anterior cingulate cortex, error detection, and the online monitoring of performance. Science 280(5364):747-749

Donahue MJ, Near J, Blicher JU, Jezzard P (2010) Baseline GABA concentration and fMRI response. Neuro Image 53(2):392-398. doi:10.1016/j.neuroimage.2010.07.017

Duncan NW, Wiebking C, Northoff G (2014) Associations of regional GABA and glutamate with intrinsic and extrinsic neural activity in humans - a review of multimodal imaging studies. Neurosci Biobehav Rev 47:36-52. doi:10.1016/j.neubiorev. 2014.07.016

Edden RA, Intrapiromkul J, Zhu H, Cheng Y, Barker PB (2012) Measuring T2 in vivo with J-difference editing: application to GABA at 3 Tesla. J Magnet Res Imag JMRI 35(1):229-234. doi:10.1002/jmri.22865

Epp AM, Dobson KS, Dozois DJ, Frewen PA (2012) A systematic meta-analysis of the Stroop task in depression. Clin Psychol Rev 32(4):316-328. doi:10.1016/j.cpr.2012.02.005

Floyer-Lea A, Wylezinska M, Kincses T, Matthews PM (2006) Rapid modulation of GABA concentration in human sensorimotor cortex during motor learning. J Neurophysiol 95(3):1639-1644. doi:10.1152/jn.00346.2005

Goto N, Yoshimura R, Moriya J, Kakeda S, Ueda N, Ikenouchi-Sugita A, Umene-Nakano W, Hayashi K, Oonari N, Korogi Y, Nakamura J (2009) Reduction of brain gamma-aminobutyric acid (GABA) concentrations in early-stage schizophrenia patients: 3 T Proton MRS study. Schizophr Res 112(1-3):192-193. doi:10.1016/j.schres.2009.04.026

Govindaraju V, Young K, Maudsley AA (2000) Proton NMR chemical shifts and coupling constants for brain metabolites. NMR Biomed 13(3):129-153

Guitart-Masip M, Economides M, Huys QJ, Frank MJ, Chowdhury R, Duzel E, Dayan P, Dolan RJ (2014) Differential, but not opponent, effects of L-DOPA and citalopram on action learning with reward and punishment. Psychopharmacology 231(5):955-966. doi:10.1007/s00213-013-3313-4

Hasler G, van der Veen JW, Tumonis T, Meyers N, Shen J, Drevets WC (2007) Reduced prefrontal glutamate/glutamine and gamma-aminobutyric acid levels in major depression determined using proton magnetic resonance spectroscopy. Arch Gen Psychiatry 64(2):193-200. doi:10.1001/archpsyc.64.2.193

Kegeles LS, Mao X, Stanford AD, Girgis R, Ojeil N, Xu X, Gil R, Slifstein M, Abi-Dargham A, Lisanby SH, Shungu DC (2012) Elevated prefrontal cortex gamma-aminobutyric acid and glutamate-glutamine levels in schizophrenia measured in vivo with proton magnetic resonance spectroscopy. Arch Gen Psychiatry 69(5):449-459. doi:10.1001/archgenpsychiatry.2011.1519

Laird AR, McMillan KM, Lancaster JL, Kochunov P, Turkeltaub PE, Pardo JV, Fox PT (2005) A comparison of label-based review and ALE meta-analysis in the Stroop task. Hum Brain Mapp 25(1):6-21. doi:10.1002/hbm.20129

Lin Y, Stephenson MC, Xin L, Napolitano A, Morris PG (2012) Investigating the metabolic changes due to visual stimulation using functional proton magnetic resonance spectroscopy at $7 \mathrm{~T}$. J Cerebral Blood Flow Metabol Off J Int Soc Cerebral Blood Flow Metabol 32(8):1484-1495. doi:10.1038/jcbfm.2012.33

Luykx JJ, Laban KG, van den Heuvel MP, Boks MP, Mandl RC, Kahn RS, Bakker SC (2012) Region and state specific glutamate downregulation in major depressive disorder: a meta-analysis of (1)H-MRS findings. Neurosci Biobehav Rev 36(1):198-205. doi:10.1016/j.neubiorev.2011.05.014

Mangia S, Tkac I, Gruetter R, Van de Moortele PF, Maraviglia B, Ugurbil K (2007) Sustained neuronal activation raises oxidative metabolism to a new steady-state level: evidence from $1 \mathrm{H}$ NMR spectroscopy in the human visual cortex. J Cerebral Blood Flow Metabol Off $\mathbf{J}$ Int Soc Cerebral Blood Flow Metabol 27(5):1055-1063. doi:10.1038/sj.jcbfm.9600401

Markram H, Toledo-Rodriguez M, Wang Y, Gupta A, Silberberg G, Wu C (2004) Interneurons of the neocortical inhibitory system. Nat Rev Neurosci 5(10):793-807. doi:10.1038/nrn1519

Mekle R, Mlynarik V, Gambarota G, Hergt M, Krueger G, Gruetter R (2009) MR spectroscopy of the human brain with enhanced signal intensity at ultrashort echo times on a clinical platform at 3T and 7T. Magnet Reson Med Off J Soc Magnet Reson Med/ 
Soc Magnet Reson Med 61(6):1279-1285. doi:10.1002/mrm. 21961

Mekle RKS, Pfeiffer H, Schubert F, Ittermann B (2014) Detection of metabolite changes in response to a varying visual stimulation paradigm using short TE $1 \mathrm{H}$ MRS at $7 \mathrm{~T}$. Proceedings of the 22nd Annual Meeting ISMRM, Milan, Italy

Mescher M, Merkle H, Kirsch J, Garwood M, Gruetter R (1998) Simultaneous in vivo spectral editing and water suppression. NMR Biomed 11(6):266-272

Meyerhoff DJ, Mon A, Metzler T, Neylan TC (2014) Cortical gamma-aminobutyric acid and glutamate in posttraumatic stress disorder and their relationships to self-reported sleep quality. Sleep 37:893-900

Michels L, Martin E, Klaver P, Edden R, Zelaya F, Lythgoe DJ, Luchinger R, Brandeis D, O'Gorman RL (2012) Frontal GABA levels change during working memory. PLoS One 7(4):e31933. doi:10.1371/journal.pone.0031933

Michels L, Schulte-Vels T, Schick M, O'Gorman RL, Zeffiro T, Hasler G, Mueller-Pfeiffer C (2014) Prefrontal GABA and glutathione imbalance in posttraumatic stress disorder: preliminary findings. Psychiatry Res 224(3):288-295. doi:10.1016/j. pscychresns.2014.09.007

Mlynarik V, Gambarota G, Frenkel H, Gruetter R (2006) Localized short-echo-time proton MR spectroscopy with full signal-intensity acquisition. Magnet Reson Med Off J Soc Magnet Reson Med/Soc Magnet Reson Med 56(5):965-970. doi:10.1002/mrm. 21043

Moeller SJ, Honorio J, Tomasi D, Parvaz MA, Woicik PA, Volkow ND, Goldstein RZ (2014) Methylphenidate enhances executive function and optimizes prefrontal function in both health and cocaine addiction. Cereb Cortex 24(3):643-653. doi:10.1093/ cercor/bhs345

Morgan PT, Pace-Schott EF, Mason GF, Forselius E, Fasula M, Valentine GW, Sanacora G (2012) Cortical GABA levels in primary insomnia. Sleep 35:806-814

Muthukumaraswamy SD, Edden RA, Jones DK, Swettenham JB, Singh KD (2009) Resting GABA concentration predicts peak gamma frequency and fMRI amplitude in response to visual stimulation in humans. Proc Natl Acad Sci USA 106(20):8356-8361. doi:10.1073/pnas.0900728106

Near J, Andersson J, Maron E, Mekle R, Gruetter R, Cowen P, Jezzard P (2013) Unedited in vivo detection and quantification of gamma-aminobutyric acid in the occipital cortex using short-TE MRS at 3 T. NMR Biomed 26(11):1353-1362. doi:10.1002/nbm. 2960

Nee DE, Wager TD, Jonides J (2007) Interference resolution: insights from a meta-analysis of neuroimaging tasks. Cognit Aff Behav Neurosci 7(1):1-17

Northoff G, Walter M, Schulte RF, Beck J, Dydak U, Henning A, Boeker H, Grimm S, Boesiger P (2007) GABA concentrations in the human anterior cingulate cortex predict negative BOLD responses in fMRI. Nat Neurosci 10(12):1515-1517. doi:10. $1038 / \mathrm{nn} 2001$

Oldfield RC (1971) The assessment and analysis of handedness: the Edinburgh inventory. Neuropsychologia 9(1):97-113

Onur OA, Piefke M, Lie CH, Thiel CM, Fink GR (2011) Modulatory effects of levodopa on cognitive control in young but not in older subjects: a pharmacological fMRI study. J Cogn Neurosci 23(10):2797-2810. doi:10.1162/jocn.2011.21603

Ordidge RJCA, Lohman JAB (1986) Image-selected in vivo spectroscopy (ISIS). A new technique for spatially selective NMR spectroscopy. J Magn Reson 66:283-294

Patel AB, de Graaf RA, Mason GF, Rothman DL, Shulman RG, Behar KL (2005) The contribution of GABA to glutamate/ glutamine cycling and energy metabolism in the rat cortex in vivo. Proc Natl Acad Sci USA 102(15):5588-5593. doi:10. 1073/pnas.0501703102

Plante DT, Jensen JE, Schoerning L, Winkelman JW (2012) Reduced gamma-aminobutyric acid in occipital and anterior cingulate cortices in primary insomnia: a link to major depressive disorder? Neuropsychopharmacol Off Publ Am Coll Neuropsychopharmacol 37(6):1548-1557. doi:10.1038/npp.2012.4

Provencher SW (1993) Estimation of metabolite concentrations from localized in vivo proton NMR spectra. Magnet Reson Med Off J Soc Magnet Reson Med/Soc Magnet Reson Med 30(6):672-679

Rosso IM, Weiner MR, Crowley DJ, Silveri MM, Rauch SL, Jensen JE (2014) Insula and anterior cingulate GABA levels in posttraumatic stress disorder: preliminary findings using magnetic resonance spectroscopy. Depress Anxiety 31:115-123

Rothman DL, Petroff OA, Behar KL, Mattson RH (1993) Localized $1 \mathrm{H}$ NMR measurements of gamma-aminobutyric acid in human brain in vivo. Proc Natl Acad Sci USA 90(12):5662-5666

Rowland LM, Kontson K, West J, Edden RA, Zhu H, Wijtenburg SA, Holcomb HH, Barker PB (2013) In vivo measurements of glutamate, GABA, and NAAG in schizophrenia. Schizophr Bull 39(5):1096-1104. doi:10.1093/schbul/sbs092

Sanacora G, Mason GF, Rothman DL, Behar KL, Hyder F, Petroff OA, Berman RM, Charney DS, Krystal JH (1999) Reduced cortical gamma-aminobutyric acid levels in depressed patients determined by proton magnetic resonance spectroscopy. Arch Gen Psychiatry 56(11):1043-1047

Sanacora G, Mason GF, Krystal JH (2000) Impairment of GABAergic transmission in depression: new insights from neuroimaging studies. Crit Rev Neurobiol 14(1):23-45

Sanacora G, Mason GF, Rothman DL, Krystal JH (2002) Increased occipital cortex GABA concentrations in depressed patients after therapy with selective serotonin reuptake inhibitors. Am J Psychiatry 159(4):663-665

Sanacora G, Mason GF, Rothman DL, Hyder F, Ciarcia JJ, Ostroff RB, Berman RM, Krystal JH (2003) Increased cortical GABA concentrations in depressed patients receiving ECT. Am J Psychiatry 160(3):577-579

Sanacora G, Gueorguieva R, Epperson CN, Wu YT, Appel M, Rothman DL, Krystal JH, Mason GF (2004) Subtype-specific alterations of gamma-aminobutyric acid and glutamate in patients with major depression. Arch Gen Psychiatry 61(7):705-713. doi:10.1001/archpsyc.61.7.705

Schaller B, Mekle R, Xin L, Kunz N, Gruetter R (2013) Net increase of lactate and glutamate concentration in activated human visual cortex detected with magnetic resonance spectroscopy at 7 tesla. J Neurosci Res 91(8):1076-1083. doi:10.1002/jnr.23194

Schaller B, Xin L, O'Brien K, Magill AW, Gruetter R (2014) Are glutamate and lactate increases ubiquitous to physiological activation? A (1)H functional MR spectroscopy study during motor activation in human brain at 7Tesla. NeuroImage 93(Pt 1):138-145. doi:10.1016/j.neuroimage.2014.02.016

Schousboe A, Westergaard N, Hertz L (1993) Neuronal-astrocytic interactions in glutamate metabolism. Biochem Soc Trans 21(1):49-53

Schubert F, Gallinat J, Seifert F, Rinneberg H (2004) Glutamate concentrations in human brain using single voxel proton magnetic resonance spectroscopy at 3 Tesla. NeuroImage 21(4):1762-1771. doi:10.1016/j.neuroimage.2003.11.014

Song X-W, Dong Z-Y, Long X-Y, Li S-F, Zuo X-N, Zhu C-Z, He Y, Yan C-G, Zang Y-F (2011) REST: a toolkit for resting-state functional magnetic resonance imaging data processing. PLoS One 6(9):e25031. doi:10.1371/journal.pone.0025031

Stagg CJ, Best JG, Stephenson MC, O'Shea J, Wylezinska M, Kincses ZT, Morris PG, Matthews PM, Johansen-Berg H (2009a) Polarity-sensitive modulation of cortical 
neurotransmitters by transcranial stimulation. J Neurosci Off $\mathbf{J}$ Soc Neurosci 29(16):5202-5206. doi:10.1523/JNEUROSCI. 4432-08.2009

Stagg CJ, Wylezinska M, Matthews PM, Johansen-Berg H, Jezzard P, Rothwell JC, Bestmann S (2009b) Neurochemical effects of theta burst stimulation as assessed by magnetic resonance spectroscopy. J Neurophysiol 101(6):2872-2877. doi:10.1152/ jn. 91060.2008

Stagg CJ, Bachtiar V, Johansen-Berg H (2011) The role of GABA in human motor learning. Curr Biol CB 21(6):480-484. doi:10. 1016/j.cub.2011.01.069

Stroop JR (1935) Studies of interference in serial verbal reactions. J Exp Psychol 18:643-662

Tkac I, Oz G, Adriany G, Ugurbil K, Gruetter R (2009) In vivo 1H NMR spectroscopy of the human brain at high magnetic fields: metabolite quantification at 4T vs. 7 T. Magnet Reson Med Off J Soc Magnet Reson Med/Soc Magn Reson Med 62(4):868-879. doi:10.1002/mrm.22086
Voon V, Reynolds B, Brezing C, Gallea C, Skaljic M, Ekanayake V, Fernandez H, Potenza MN, Dolan RJ, Hallett M (2010) Impulsive choice and response in dopamine agonist-related impulse control behaviors. Psychopharmacology 207(4):645-659. doi:10.1007/s00213-009-1697-y

Wang XJ (2010) Neurophysiological and computational principles of cortical rhythms in cognition. Physiol Rev 90(3):1195-1268. doi:10.1152/physrev.00035.2008

Winterer G, Weinberger DR (2004) Genes, dopamine and cortical signal-to-noise ratio in schizophrenia. Trends Neurosci 27(11):683-690. doi:10.1016/j.tins.2004.08.002

Yoon JH, Maddock RJ, Rokem A, Silver MA, Minzenberg MJ, Ragland JD, Carter CS (2010) GABA concentration is reduced in visual cortex in schizophrenia and correlates with orientationspecific surround suppression. J Neurosci Off J Soc Neurosci 30(10):3777-3781. doi:10.1523/JNEUROSCI.6158-09.2010 